\title{
Liraglutide changes body composition and lowers added sugar intake in overweight persons with insulin pump-treated type 1 diabetes
}

\author{
Signe Schmidt MD $D^{1,2,3,4}$ ๑ | Christian S. Frandsen $M^{2,5}$ | \\ Thomas F. Dejgaard MD ${ }^{1,6}$ () | Dorte Vistisen $\mathrm{MSc}^{1,7}$ | Thórhallur Halldórsson $\mathrm{MSc}^{8}$ | \\ Sjudur F. Olsen $\mathrm{MSc}^{9}$ ｜ Jens-Erik B. Jensen $\mathrm{MD}^{2}$ ｜ Sten Madsbad MD² ${ }^{2}$ | \\ Henrik U. Andersen $M^{1} \quad$ Kirsten Nørgaard MD ${ }^{1,2,10}$
}

${ }^{1}$ Clinical Research, Steno Diabetes Center Copenhagen, Gentofte, Denmark

${ }^{2}$ Department of Endocrinology, Copenhagen University Hospital Hvidovre, Hvidovre,

Denmark

${ }^{3}$ Danish Diabetes Academy, Odense, Denmark

${ }^{4}$ Department of Clinical Pharmacology, Bispebjerg and Frederiksberg University Hospital, Copenhagen, Denmark

${ }^{5}$ Department of Obstetrics and Gynecology,

Rigshospitalet, Glostrup, Denmark

${ }^{6}$ Centre for Clinical Metabolic Research, Copenhagen University Hospital Gentofte, Gentofte, Denmark

${ }^{7}$ Department of Public Health, University of Copenhagen, Copenhagen, Denmark

${ }^{8}$ Faculty of Food Science and Nutrition, School of Health Sciences, University of Iceland,

Reykjavik, Iceland

${ }^{9}$ Department of Epidemiology Research, Statens Serum Institut, Copenhagen, Denmark

${ }^{10}$ Department of Clinical Medicine, Faculty of Health and Medical Sciences, University of Copenhagen, Copenhagen, Denmark

\section{Correspondence}

Signe Schmidt, Steno Diabetes Center

Copenhagen, Niels Steensens Vej 2-4, 2820

Gentofte, Denmark.

Email: signe.schmidt@regionh.dk

\begin{abstract}
Aims: To present secondary outcome analyses of liraglutide treatment in overweight adults with insulin pump-treated type 1 diabetes (T1D), focusing on changes in body composition and dimensions, and to evaluate changes in food intake to identify potential dietary drivers of liraglutide-associated weight loss.

Materials and methods: A 26-week randomized placebo-controlled study was conducted to investigate the efficacy and safety of liraglutide $1.8 \mathrm{mg}$ daily in 44 overweight adults with insulin pump-treated T1D and glucose levels above target, and demonstrated significant glycated haemoglobin (HbA1c)- and body weight-reducing effects. For secondary outcome analysis, dual X-ray absorptiometry scans were completed at Weeks 0 and 26, and questionnaire-based food frequency recordings were obtained at Weeks 0,13 and 26 to characterize liraglutide-induced changes in body composition and food intake.

Results: Total fat and lean body mass decreased in liraglutide-treated participants (fat mass $-4.6 \mathrm{~kg}[95 \%$ confidence interval $\{\mathrm{Cl}\}-5.7 ;-3.5], P<0.001$; lean mass $-2.5 \mathrm{~kg}[95 \% \mathrm{Cl}-3.2 ;-1.7], \mathrm{P}<0.001$ ), but remained stable in placebo-treated participants (fat mass $-0.3 \mathrm{~kg}[95 \% \mathrm{Cl}-1.3 ; 0.8], P=0.604$; lean mass $0.0 \mathrm{~kg}[95 \%$ $\mathrm{Cl}-0.7 ; 0.7$ ]; $P=0.965$ [between-group $P$ values $<0.001$ ]). Participants reduced their energy intake numerically more in the liraglutide arm $(-1.1 \mathrm{MJ}[95 \% \mathrm{Cl}-2.0 ;-0.02]$, $P=0.02)$ than in the placebo arm $(-0.9 \mathrm{MJ}[95 \% \mathrm{Cl}-2.0 ; 0.1], P=0.22)$, but the between-group difference was statistically insignificant $(P=0.42)$. However, energy derived from added sugars decreased by $27 \%$ in the liraglutide arm compared with an increase of $14 \%$ in the placebo arm $(P=0.004)$.

Conclusions: Liraglutide lowered fat and lean body mass compared with placebo. Further, liraglutide reduced intake of added sugars. However, no significant difference in total daily energy intake was detected between liraglutide- and placebotreated participants.
\end{abstract}




\section{KEYWORDS}

body composition, food frequency, insulin pump therapy, liraglutide, type 1 diabetes, weight loss

\section{1 | INTRODUCTION}

A few decades ago, type 1 diabetes (T1D) could be discriminated from other types of diabetes based on phenotype. Most people with T1D were lean or underweight, but today, more than half of the adult population with T1D is overweight or obese. ${ }^{1,2}$ Further, despite increasing use of advanced diabetes technologies, the recommended glycaemic goal of glycated haemoglobin (HbA1c) $<53 \mathrm{mmol} / \mathrm{mol}(7.0 \%)$ is achieved by only every fifth person with T1D. ${ }^{3}$ This means that most T1D treatment should not only address glucose management but also weight management to minimize the risk of diabetes- and obesityassociated morbidity and mortality. ${ }^{4-8}$

A number of add-ons to insulin with the potential to improve glucose levels while sustaining or even reducing body weight has been investigated in open- and closed-loop therapies. ${ }^{9-12}$ In particular, the glucagon-like peptide-1 (GLP-1) receptor agonist liraglutide has been the target of study in clinical trials in different subgroups of persons with T1D. ${ }^{13-21}$ A recent meta-analysis of randomized controlled trials of at least 4 weeks' duration showed that when liraglutide is added to insulin therapy, persons with T1D on average achieve a decrease in $\mathrm{HbA} 1 \mathrm{c}$ of $2 \mathrm{mmol} / \mathrm{mol}(0.2 \%)$ and a $3.85-\mathrm{kg}$ weight loss. ${ }^{12}$

Currently, liraglutide is only approved for the treatment of type 2 diabetes, overweight and obesity, and its off-label use for the management of T1D is relatively limited. ${ }^{2}$ Nevertheless, the combination of even a modest reduction of $\mathrm{HbA} 1 \mathrm{c}$ and a moderate weight loss makes liraglutide an attractive therapeutic option for persons with T1D who struggle with glucose values and a body weight above the recommended levels despite dietary guidance, diabetes education and diabetes technology use.

In a previously published 26-week randomized placebo-controlled study of efficacy and safety of liraglutide in overweight adults with insulin pump-treated T1D and glucose levels above target, the Lira Pump trial, we found greater reductions in $\mathrm{HbA1c}(-7.3 \mathrm{mmol} / \mathrm{mol}$ [-0.7\%]) and weight $(-6.3 \mathrm{~kg})$ than have been observed in the general T1D population. ${ }^{18}$

In the present paper, we report on secondary outcome analyses, focusing on changes in body composition and dimensions, and we evaluate changes in food intake to identify potential dietary drivers of the liraglutide-associated weight loss.

\section{2 | MATERIALS AND METHODS}

\section{1 | Study design}

The present study is a substudy of the Lira Pump Trial, a 26-week, randomized, double-blind, placebo-controlled, dual-centre study of the effects of liraglutide $1.8 \mathrm{mg}$ once daily in adults with insulin pump-treated T1D who were overweight or obese and had an HbA1c above the recommended level. Details of the primary study have been published elsewhere. ${ }^{18}$

The study was undertaken at Copenhagen University Hospital Hvidovre and Steno Diabetes Center Copenhagen in collaboration with the National Serum Institute (Statens Serum Institut) in Denmark. The study protocol was approved by the Scientific-Ethical Committee of the Capital Region of Denmark (H-3-2014-094), the Danish Medicines Authority (EudraCT 2014-002285-76), and the Danish Data Protection Agency (AHH-2015-002/03497), conducted under the surveillance of the Good Clinical Practice Unit at Copenhagen University Hospital, and registered at ClinicalTrials.gov (NCT02351232).

The study included adults (aged 18-70 years) with T1D and insulin pump use for $\geq 1$ year, body mass index $(B M I)>25 \mathrm{~kg} / \mathrm{m}^{2}$, $\mathrm{HbA} 1 \mathrm{c}>58 \mathrm{mmol} / \mathrm{mol}$ (7.5\%), and consistent use of carbohydrate counting and entry of carbohydrates in the insulin pump bolus calculator. Persons with gastroparesis, inflammatory bowel disease, renal dysfunction (estimated glomerular filtration rate $<60 \mathrm{~mL} / \mathrm{min} / 1.73 \mathrm{~m}^{2}$ ), history of pancreatitis, cancer or other major medical or psychological condition, and concurrent use of corticosteroids or antidiabetic medicine other than insulin were ineligible for participation.

Participants were randomized in a 1:1 ratio to liraglutide or placebo. Study personnel and participants were blinded to the allocation. Liraglutide and placebo injection pens that were indistinguishable from one another were provided by the manufacturer (Novo Nordisk A/S, Måløv, Denmark). The titration methods for the two solutions were identical and as follows: $0.6 \mathrm{mg}$ liraglutide/ $0.1 \mathrm{~mL}$ placebo was injected once daily for the first week, the second week the dose was increased to $1.2 \mathrm{mg}$ liraglutide $/ 0.2 \mathrm{~mL}$ placebo, and finally in the third week of study participation the dose was increased to $1.8 \mathrm{mg}$ liraglutide $/ 0.3 \mathrm{~mL}$ placebo. The intervals between dose increments could be extended at the discretion of the investigator in case of pronounced gastrointestinal side effects, and if $1.8 \mathrm{mg}$ per day was not tolerated, participants could stay on $1.2 \mathrm{mg}$ per day for the duration of the study.

Participants' insulin pump therapy with rapid-acting insulin analogue was continued during the study and managed according to usual standards based on blood glucose monitoring or continuous glucose monitoring (CGM) values. No dietary guidance or specific guidelines for managing hypoglycaemia were provided. However, at study start-as a safe practice to reduce the risk of hypoglycaemia in participants receiving liraglutide-all insulin basal rates were reduced by $10 \%$ and insulin bolus doses were lowered by increasing correction factors and insulinto-carbohydrate ratios by $15 \%$. Insulin pump settings were subsequently adjusted to appropriate levels by the participants' themselves or by the study personnel at the first telephone contact 1 week later. 
Study participation included a screening visit, four visits at the outpatient diabetes clinics (Weeks 0, 3, 13 and 26), and one telephone consultation (Week 1). Insulin pump data were uploaded, evaluated, and adjusted if needed at each contact. Six days of CGM were undertaken with blinded devices (iPro2 [Medtronic, Northridge, California]) four times during the study (Weeks 0, 3, 13 and 26).

The primary study endpoint was difference in change in $\mathrm{HbA} 1 \mathrm{c}$ from Week 0 to Week 26 between participants treated with liraglutide and placebo. Differences in change in anthropometry, body composition, self-reported food intake and physical activity level, which are reported in this paper, were among the predefined secondary study endpoints.

\section{2 | Anthropometry}

At Week 0 , height, body weight, waist circumference and hip circumference were measured according to World Health Organization recommendations. ${ }^{22}$ The latter three were repeated at Weeks 3, 13 and 26. Subsequently, waist-to-hip ratio and BMI were calculated for each of the timepoints.

\section{3 | Body composition}

At Week 0 and Week 26, fat and lean body mass were determined by dual X-ray absorptiometry (DXA) scans using a Hologic Discovery A (Hvidovre Hospital: S/N 83487; Steno Diabetes Center Copenhagen: S/N 82800) DXA scanner (Hologic Inc., Marlborough, Massachusetts). Participants were scanned in a fasting state, and during the procedure insulin pumps and continuous glucose monitors were disconnected and removed from the body to avoid artefacts. The android region was defined as the area of the abdomen from a line joining the two superior iliac crests and extended cranially for $20 \%$ of the distance to the base of the skull. The gynoid region was defined as the portion of the legs from the greater femoral trochanter, extending caudally to mid-thigh.

\subsection{Food frequency questionnaire}

Participants filled out a web-based food frequency questionnaire (FFQ) with a 12-week recall period at Week 0, Week 13 and Week 26. The FFQ was a modified version of the 360-item FFQ used for the Danish National Birth Cohort, which has been validated against 7-day food diaries and selected biomarkers. ${ }^{23-25}$ The modifications included conversion from paper to electronic format and updating the questionnaire to cover temporal changes in dietary habits since the initial development for the Danish National Birth Cohort. The amount of foods consumed were then quantified based on reported frequency of consumption and assumptions on standard portion sizes. The amount of nutrients consumed was quantified using the Danish Food Composition table. $^{26}$

\subsection{Physical activity assessment}

Participants were asked at Week 0, Week 13 and Week 26 how often they performed exercise causing sweating and an increase in heart and breathing rates for more than 30 minutes. The scale used had five levels: (a) daily; (b) 3 to 4 times per week; (c) 1 to 2 times per week; (d) rarely; and (e) never.

\subsection{Statistical analyses}

Measures of body composition, clinical biomarkers and dietary intake of participants at baseline were described using mean and standard deviation or median and interquartile range. Changes over time (Week 0-Week 13-Week 26) within groups (t-test) and between groups (F-test) were modelled using linear mixed-effects models. Changes in physical activity were assessed using Fisher's exact test. All analyses were performed as an intention-to-treat analysis, that is, including all available data for all participants, completers as well as non-completers. The statistical significance level was set at $P<0.05$. Statistical analyses were conducted in SAS version 9.2.

\section{3 | RESULTS}

In February 2015 to April 2016, 171 adults with insulin pump-treated T1D were assessed for eligibility. Of these, 54 were screened, and 44 of them met the inclusion criteria and were randomized to either liraglutide or placebo treatment. Of the 22 participants assigned to the liraglutide study arm, three persons withdrew because of breast cancer, multiple sclerosis and gastrointestinal side effects, respectively. All 22 participants assigned to the placebo study arm completed the study.

Participant characteristics including a summary of primary and selected secondary study endpoints, which have been previously reported, are given in Table $1 .{ }^{18}$ In brief, changes in $\mathrm{HbA} 1 \mathrm{c}$ were significantly different between participants treated with liraglutide $-5 \mathrm{mmol} / \mathrm{mol}(-0.5 \%)$ and participants treated with placebo $2 \mathrm{mmol} /$ $\mathrm{mol}(0.2 \% ; P<0.001)$. This was reflected by a significant difference between the two groups in time spent with glucose values in the range 4.0 to $10.0 \mathrm{mmol} / \mathrm{L}(P=0.044)$ : participants receiving liraglutide increased time in range by $4.6 \%$, that is, by 66 minutes, whereas participants receiving placebo reduced time in range by $4.8 \%$, that is, by 69 minutes. The total daily insulin dose decreased by $4.9 \mathrm{IU}$ in the liraglutide study arm and increased by $2.8 \mathrm{IU}$ in the placebo study arm ( $P=0.008$ for between-group difference). This was mainly driven by a difference in change in total daily bolus insulin dose. Finally, there was a significant 6.8-kg decrease in body weight in participants receiving liraglutide, whereas there was no change in body weight in those receiving placebo $(P<0.001$ for between-group difference).

The incidence of gastrointestinal symptoms was higher in the liraglutide study arm than in the placebo arm, with nausea being the 
TAB LE 1 Participant baseline characteristics and changes within and between groups after 26 weeks of treatment with liraglutide or placebo

\begin{tabular}{|c|c|c|c|c|c|c|c|}
\hline & \multicolumn{3}{|l|}{ Liraglutide } & \multicolumn{3}{|l|}{ Placebo } & \multirow{2}{*}{$\begin{array}{l}\Delta \text { Liraglutide } \\
\text { vs } \Delta \text { Placebo } \\
P \text { value }\end{array}$} \\
\hline & Baseline & Change & $P$ value & Baseline & Change & $P$ value & \\
\hline Randomized/female sex, $\mathrm{N}$ & $22 / 15$ & & & $22 / 15$ & & & \\
\hline Age, years & $54(37-61)$ & & & $45(34-52)$ & & & \\
\hline Type 1 diabetes, years & $21(15-34)$ & & & $20(15-35)$ & & & \\
\hline Stimulated C-peptide, pmol/L & $9(7-18)$ & & & $9(6-12)$ & & & \\
\hline rt-CGM use, $n$ & 10 & & & 6 & & & \\
\hline Body mass index, $\mathrm{kg} / \mathrm{m}^{2}$ & $30.2 \pm 2$ & $-2.4(-2.9 ;-1.9)$ & $<0.001$ & $29 \pm 3$ & $-0.2(-0.7 ; 0.3)$ & 0.487 & $<0.001$ \\
\hline $\mathrm{HbA} 1 \mathrm{c}, \mathrm{mmol} / \mathrm{mol}$ & $66 \pm 6$ & $-5(-8 ;-2)$ & $<0.001$ & $66 \pm 6$ & $2(0 ; 5)$ & 0.058 & $<0.001$ \\
\hline $\mathrm{HbA1c}, \%$ & $8.2 \pm 0.5$ & $-0.5(-0.7 ;-0.2)$ & $<0.001$ & $8.1 \pm 0.5$ & $0.2(0.0 ; 0.4)$ & 0.058 & $<0.001$ \\
\hline Percentage of time at $<4.0 \mathrm{mmol} / \mathrm{L}$ & $3.2 \pm 3.5$ & $2.5(0.1 ; 5.0)$ & 0.044 & $3.5 \pm 3.6$ & $2.1(-0.2 ; 4.4)$ & 0.077 & 0.787 \\
\hline Percentage of time at 4.0 to $10.0 \mathrm{mmol} / \mathrm{L}$ & $51.3 \pm 12.7$ & $4.6(-2.1 ; 11.3)$ & 0.178 & $48.6 \pm 12.4$ & $-4.8(-11.0 ; 1.5)$ & 0.133 & 0.044 \\
\hline Percentage of time at $>10.0 \mathrm{mmol} / \mathrm{L}$ & $45.9 \pm 14.0$ & $-7.2(-14.6 ; 0.1)$ & 0.053 & $47.9 \pm 13.3$ & $2.7(-4.1 ; 9.5)$ & 0.441 & 0.052 \\
\hline \multicolumn{8}{|l|}{ Insulin pump data } \\
\hline Total daily insulin dose, $U$ & $48 \pm 15$ & $-4.9(-9.1 ;-0.7)$ & 0.021 & $54 \pm 19$ & $2.8(-1.1 ; 6.7)$ & 0.164 & 0.008 \\
\hline Total daily basal insulin dose, $U$ & $25 \pm 9$ & $-1.8(-3.3 ;-0.3)$ & 0.017 & $28 \pm 7$ & $-0.5(-1.9 ; 0.9)$ & 0.473 & 0.214 \\
\hline Total daily bolus insulin dose, $U$ & $23 \pm 9$ & $-3.1(-6.8 ; 0.7)$ & 0.113 & $26 \pm 14$ & $3.3(-0.3 ; 6.9)$ & 0.069 & 0.016 \\
\hline Total daily carbohydrate intake, $\mathrm{g}$ & $119 \pm 39$ & $-6(-28 ; 16)$ & 0.578 & $137 \pm 71$ & $14(-7 ; 34)$ & 0.184 & 0.188 \\
\hline
\end{tabular}

Note: Baseline data are mean \pm standard deviation and median (interquartile range). Estimated changes within groups are mean ( $95 \%$ confidence interval). $P$ values reflect change over time within each treatment group and difference in change over time between the liraglutide and placebo groups. Time at $<4.0 \mathrm{mmol} / \mathrm{L}=$ time spent with sensor glucose values $<4.0 \mathrm{mmol} / \mathrm{L}$; time at 4.0 to $10.0 \mathrm{mmol} / \mathrm{L}=$ time spent with sensor glucose values; time at $>10.0 \mathrm{mmol} / \mathrm{L}=$ time spent with sensor glucose values $>10.0 \mathrm{mmol} / \mathrm{L}$.

Abbreviation: rtCGM, real-time continuous glucose monitoring.

FIGURE 1 Fat and lean body mass determined by dual-energy $\mathrm{X}$-ray absorptiometry at Week 0 and Week 26 in participants treated with liraglutide and placebo. NS, not significant

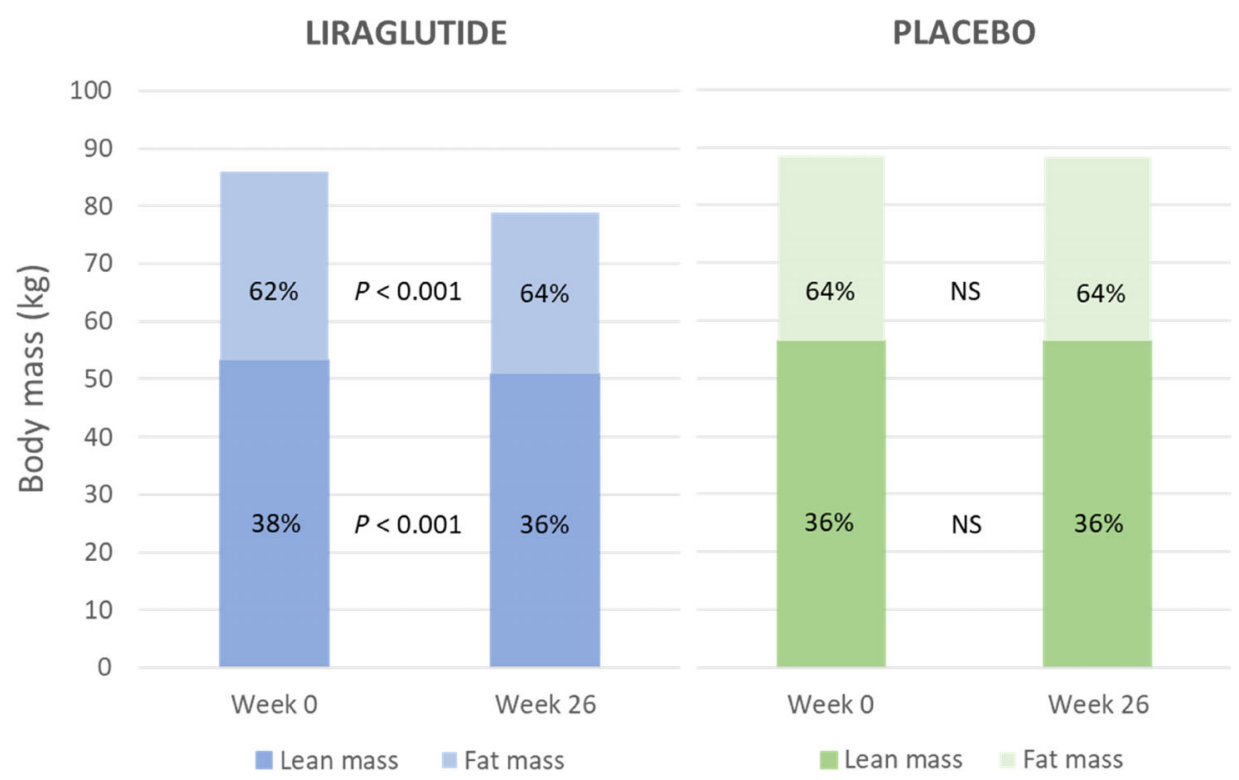

most prevalent. Five persons (23\%) receiving liraglutide treatment had to deviate from the titration plan described in the study protocol and postpone the dose escalation from 1.2 to $1.8 \mathrm{mg}$ liraglutide because of gastrointestinal side effects. One of the five persons did not tolerate the end dose and had to stay on 1.2-mg liraglutide daily for the duration of the study. 
TAB LE 2 Body composition and anthropometric data for participants treated with liraglutide compared with placebo

\begin{tabular}{|c|c|c|c|c|c|c|c|c|}
\hline & \multicolumn{3}{|l|}{ Liraglutide } & \multicolumn{3}{|l|}{ Placebo } & \multicolumn{2}{|c|}{$\begin{array}{l}\Delta \text { Liraglutide vs. } \\
\Delta \text { Placebo }\end{array}$} \\
\hline & Baseline & Change & $P$ value & Baseline & Change & $P$ value & $P$ value & $P$ value* \\
\hline Total mass, kg & $85.9 \pm 10.0$ & $-7.0(-8.5 ;-5.5)$ & $<0.001$ & $88.6 \pm 12.9$ & $-0.3(-1.7 ; 1.1)$ & 0.687 & $<0.001$ & $<0.001$ \\
\hline Total lean mass, kg & $53.3 \pm 9.5$ & $-2.5(-3.2 ;-1.7)$ & $<0.001$ & $56.4 \pm 12.7$ & $0.0(-0.7 ; 0.7)$ & 0.965 & $<0.001$ & $<0.001$ \\
\hline Trunk lean mass, kg & $26.2 \pm 4.8$ & $-1.3(-1.7 ;-0.9)$ & $<0.001$ & $27.7 \pm 5.8$ & $-0.1(-0.5 ; 0.3)$ & 0.556 & $<0.001$ & $<0.001$ \\
\hline Trunk fat mass, kg & $14.9 \pm 3.5$ & $-2.4(-3.0 ;-1.8)$ & $<0.001$ & $14.9 \pm 3.1$ & $-0.2(-0.8 ; 0.3)$ & 0.430 & $<0.001$ & $<0.001$ \\
\hline Android lean mass, $\mathrm{kg}$ & $4.2 \pm 0.9$ & $-0.2(-0.3 ;-0.1)$ & $<0.001$ & 4.30 .8 & $0.0(-0.1 ; 0.1)$ & 0.471 & 0.003 & 0.002 \\
\hline Android fat mass, $\mathrm{kg}$ & $2.8 \pm 0.8$ & $-0.5(-0.7 ;-0.4)$ & $<0.001$ & $2.7 \pm 0.7$ & $0.0(-0.1 ; 0.2)$ & 0.778 & $<0.001$ & $<0.001$ \\
\hline Gynoid lean mass, kg & $8.4 \pm 1.5$ & $-0.5(-0.6 ;-0.3)$ & $<0.001$ & 8.91 .9 & $-0.1(-0.2 ; 0.1)$ & 0.323 & 0.001 & $<0.001$ \\
\hline \multicolumn{9}{|l|}{ Anthropometric measurements } \\
\hline Waist circumference, $\mathrm{cm}$ & $98.6 \pm 9.8$ & $-7.6(-9.7 ;-5.5)$ & $<0.001$ & $98.0 \pm 10.1$ & $-1.1(-3.0 ; 0.9)$ & 0.272 & $<0.001$ & $<0.001$ \\
\hline Hip circumference, $\mathrm{cm}$ & $110.6 \pm 6.7$ & $-4.1(-5.5 ;-2.6)$ & $<0.001$ & $111.5 \pm 5.5$ & $0.1(-1.2 ; 1.5)$ & 0.845 & $<0.001$ & $<0.001$ \\
\hline Waist / hip ratio & $0.89 \pm 0.10$ & $-0.04(-0.06 ;-0.02)$ & $<0.001$ & $0.88 \pm 0.09$ & $-0.01(-0.03 ; 0.01)$ & 0.228 & 0.023 & 0.018 \\
\hline
\end{tabular}

Note: Baseline data are mean \pm standard deviation. Estimated changes within groups are mean ( $95 \%$ confidence interval). $P$ values reflect change over time within each treatment group and difference in change over time between the liraglutide and placebo groups.

${ }^{*}$ Adjusted for sex and age. Android region: the area of the abdomen from a line joining the two superior iliac crests and extended cranially for $20 \%$ of the distance to the base of the skull. Gynoid region: the portion of the legs from the greater femoral trochanter, extending caudally to mid-thigh.

TAB LE 3 Food recordings for participants treated with liraglutide compared with placebo

\begin{tabular}{|c|c|c|c|c|c|c|c|}
\hline & \multicolumn{3}{|l|}{ Liraglutide } & \multicolumn{3}{|l|}{ Placebo } & \multirow{2}{*}{$\begin{array}{l}\Delta \text { Liraglutide } \\
\text { vs. } \Delta \text { Placebo } \\
P \text { value }\end{array}$} \\
\hline & Baseline & Change & $P$ value & Baseline & Change & $P$ value & \\
\hline Energy, MJ & $7.1 \pm 2.9$ & $-1.1(-2.0 ;-0.02)$ & 0.02 & $7.5 \pm 2.6$ & $-0.9(-2.0 ; 0.1)$ & 0.22 & 0.42 \\
\hline Protein, \%E & $18.0 \pm 3.0$ & $1.2(-0.3 ; 2.7)$ & 0.12 & $18.7 \pm 2.7$ & $1.0(-1.1 ; 3.1)$ & 0.33 & 0.24 \\
\hline Total fat, \%E & $34.3 \pm 4.7$ & $1.2(-1.7 ; 4.1)$ & 0.40 & $36.7 \pm 6.4$ & $-1.1(-3.5 ; 1.2)$ & 0.33 & 0.37 \\
\hline PUFA & $5.8 \pm 0.9$ & $-0.3(-0.6 ; 0.1)$ & 0.15 & $6.1 \pm 1.4$ & $-0.1(-0.6 ; 0.4)$ & 0.66 & 0.43 \\
\hline Carbohydrates, \%E & $42.8 \pm 7.8$ & $-3.4(-7.2 ; 0.5)$ & 0.08 & $41.5 \pm 7.4$ & $1.1(-1.1 ; 3.4)$ & 0.32 & 0.14 \\
\hline Carbohydrates, g/d & $175 \pm 95$ & $-42(-78 ;-5)$ & 0.03 & $182 \pm 85$ & $-24(-49 ; 0.4)$ & 0.05 & 0.21 \\
\hline Added sugars, \%E & $4.6 \pm 2.7$ & $-1.2(-2.3 ; 0.0)$ & 0.05 & $5.1 \pm 4.0$ & $1.0(-0.8 ; 2.2)$ & 0.35 & 0.004 \\
\hline
\end{tabular}

Note: Baseline data are mean \pm standard deviation. Estimated changes within groups are mean $(95 \%$ confidence interval). $P$ values reflect change over time within each treatment group and difference in change over time between the liraglutide and placebo groups.

Abbreviations: MUFA, monounsaturated fatty acids; PUFA, polyunsaturated fatty acids; SFA, saturated fatty acids.

The DXA scans showed that the significant weight loss observed in the liraglutide study arm was the result of significant reductions in fat $(-4.6 \mathrm{~kg})$ as well as fat-free mass $(-2.5 \mathrm{~kg})$, whereas no changes in either of the two tissue types were observed in the placebo study arm (Figure 1). Further details of the DXA scan results are given in Table 2.
Waist circumference and hip circumference decreased significantly in participants treated with liraglutide $(-7.6$ and $-4.1 \mathrm{~cm}$, respectively), but the former more than the latter which is why the waist-hip ratio also decreased. There were no changes in waist and hip circumference in the placebo-treated participants (Table 2). 

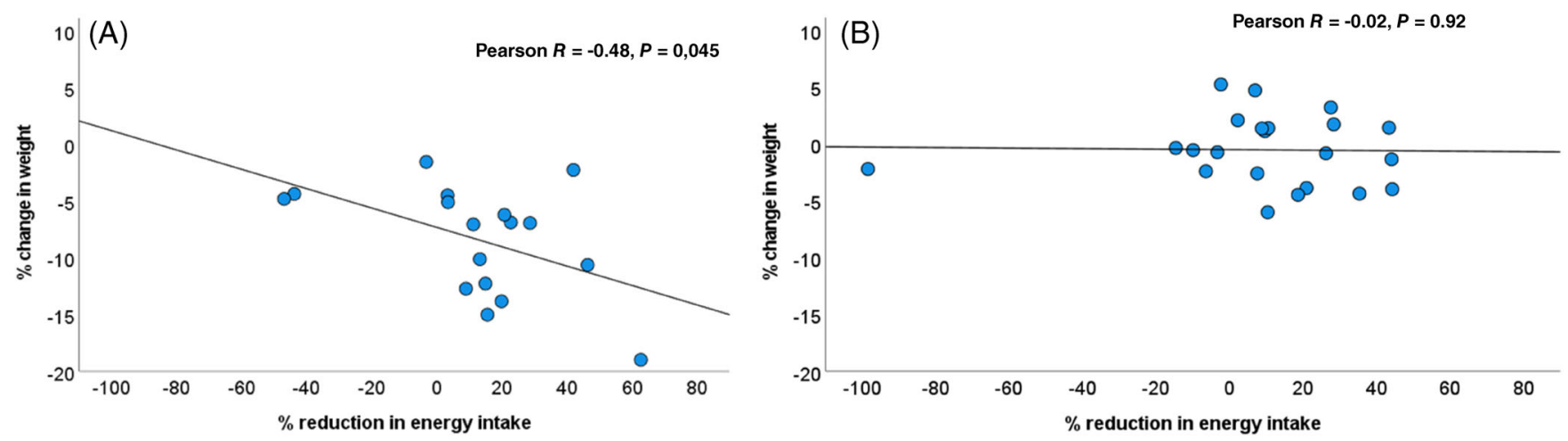

FIGURE 2 Scatter plots of relative change in weight versus relative reduction in energy intake from Week 0 to Week 26 . A, Liraglutidetreated participants. B, Placebo-treated participants
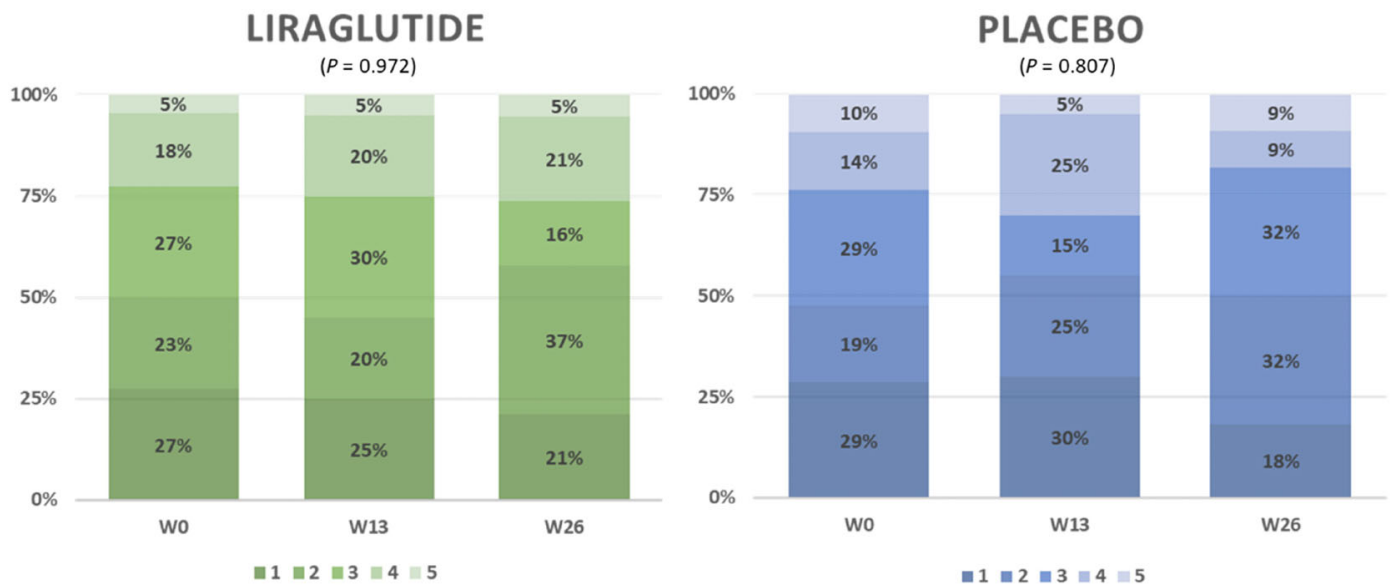

FIGURE 3 Participant-reported exercise level. Exercise causing sweating and an increase in heart and breathing rate $>30$ min: $1=$ daily; $2=3$ to 4 times per week; $3=1$ to 2 times per week; $4=$ rarely; $5=$ never. $P$ values reflect changes over time within each treatment group. W, week

Baseline energy intake (mean \pm SD) was $7.1 \pm 2.9 \mathrm{MJ}$ and $7.5 \pm 2.6 \mathrm{MJ}$ in the liraglutide and placebo group, respectively (Table 3). During the study period, participants in the liraglutide group reduced their energy intake significantly $-1.1 \mathrm{MJ}(95 \% \mathrm{Cl}-2.0$; -0.02), while a numerically similar but statistically insignificant decrease was observed in the placebo group $-0.9 \mathrm{MJ}(95 \% \mathrm{Cl}-2.0$; $0.1)$. There was no difference in change in energy intake between the groups $(P=0.42)$. In liraglutide-treated persons, each 1-MJ decrease in daily energy intake was found to be associated with a $2.0-\mathrm{kg}$ weight loss (95\% $\mathrm{Cl} 3.1 ; 0.9 ; P=0.002)$ after adjustment for baseline energy intake, age and sex (Figure 2). In contrast, in placebo-treated persons, a more modest and nonsignificant weight loss of $0.2 \mathrm{~kg}(95 \% \mathrm{Cl}-0.6$; $0.9 ; P=0.66$ ) was observed for each $1-\mathrm{MJ}$ decrease in daily energy intake. The only significantly different change in food intake over time between the two groups was observed for added sugars $(P=0.004)$ where intake decreased in the liraglutide group ( $1 \%$ of energy), while intake increased ( $\sim 1 \%$ of energy) in the placebo group. Further details of the FFQ recordings are given in Table 3.
The majority of participants reported being regularly physically active. There were no significant changes in participant-reported activity level in the two study arms throughout the study (Figure 3).

\section{DISCUSSION}

In this secondary endpoint analysis of the 26-week randomized placebo-controlled Lira Pump Trial in overweight adults with insulin pump-treated T1D and glucose levels above target, we demonstrated that the observed liraglutide-induced weight loss was the result of significant reductions in total fat and lean body mass. Both gynoid and android fat mass decreased, but the former proportionally more than the latter, which led to a significant albeit small reduction in the ratio between the two. In parallel, waist-to-hip ratio decreased. The participants' level of physical activity did not change throughout the study. We were unable to identify dietary changes that could explain the pronounced weight loss, however, we did register a significant 
reduction in the intake of added sugars when comparing the liraglutide arm with the placebo arm.

The present study is not the first to report changes in body composition in persons with T1D treated with $1.8 \mathrm{mg}$ liraglutide. ${ }^{19,21}$ Interestingly, our results differ from the results of the two previously published studies in the sense that, in addition to significant reductions in fat mass, we document clinically and statistically significant reductions in lean mass in the liraglutide arm. The ratio between fat and lean mass reductions in the present study (1.8:1.0) instead resembles what has been reported in studies of liraglutide-treated persons with type 2 diabetes. ${ }^{27}$ The most recently published of the two T1D studies has many similarities with the present study, including study design (26-week parallel intervention and placebo arms) and method of assessment of changes in body composition (DXA scans). ${ }^{19}$ However, there are also several differences between the two which might explain the differences in outcomes, such as method of insulin therapy (100\% insulin pump use vs. $68 \%$ ) and $\mathrm{BMI}$ at baseline $\left(30.2 \mathrm{vs} .33 .3 \mathrm{~kg} / \mathrm{m}^{2}\right) .{ }^{19}$ In the present study we specifically assessed changes in exercise level as a potential contributor to change in tissue mass, but we found no indication of such an association.

It can be debated whether the body composition changes observed in liraglutide-treated participants in the present study should be characterized as beneficial. There is an increasing body of evidence suggesting that losses of fat mass and lean body mass may have oppositely operating effects on mortality-while a reduction in body fat may reduce mortality, a reduction in lean body mass may increase it. $^{28,29}$ In this case it would mean that the significant loss of lean body mass could negatively affect health. On the other hand, the lowering of BMI, waist circumference, waist-to-hip ratio, and android fat-togynoid fat ratio all contribute to a more favourable cardiometabolic risk profile. ${ }^{30-32}$ Finally, from a user perspective, the visible changes in body dimensions were probably viewed as positive, with impact on both physical and emotional well-being. ${ }^{33}$

Dietary changes as an explanatory cause of liraglutide-induced weight loss have not been previously studied in persons with T1D FFQ scores at Week 0 indicated that, in the 12 weeks prior to study entry, participants' daily energy intake was lower than would be expected for weight maintenance $(\approx 9.0 \mathrm{KJ})$. Possible explanations for this could be that these overweight and obese persons had already made attempts to lose weight through dietary restrictions, alternatively that they were underreporting their food intake. ${ }^{34}$ During the study, there was a trend toward further reductions in participantreported energy intake. The magnitude of the change in energy intake was not statistically significant between the two study arms, although numerically greater in the liraglutide-treated arm. The absence of a difference in energy intake between liraglutide- and placebo-treated participants is somewhat surprising because GLP-1-based therapies are known to reduce food intake, appetite and hunger and to promote fullness and satiety. ${ }^{35,36}$ Further, studies of GLP-1-based therapies in persons with type 2 diabetes have reported reduced food intake when assessed by ad libitum meals. ${ }^{37,38}$ In the present study, the association between energy intake at Week 26 and weight loss in the liraglutide arm suggests that absolute energy intake may, at least partly, explain the observed weight loss. However, lack of significant difference in energy intake between liraglutide- and placebo-treated participants complicates this interpretation. Nevertheless, our results point toward a potentiating effect of liraglutide and energy restriction, that is, those who limit their energy intake the most achieve the greatest weight loss during liraglutide treatment.

Although FFQ responses did not show significant changes between study arms neither in total energy intake nor in macronutrient intake, they did show a difference between liraglutide- and placebo-treated participants in terms of intake of added sugars. Added sugars contributed little to the overall energy intake; however, they may have impacted glucose levels. Thus, the reduced intake of added sugars in the liraglutide arm could, at least partly, explain the previously reported diminished insulin needs and improvements in $\mathrm{HbA1c}$ and time spent with continuous glucose monitoring values in the range 4.0 to $10.0 \mathrm{mmol} / \mathrm{L}{ }^{18,39}$ Further, the reductions in daily insulin dose may have contributed to the weight loss. ${ }^{40}$ Animal studies and some human studies have suggested that treatment with GLP-1 receptor agonists alters individual food preferences and consequently dietary macronutrient composition. ${ }^{38,41,42}$ However, further investigations of this association and the potential underlying central and/or peripheral mechanisms are needed to draw firm conclusions. ${ }^{35}$

The present study has both strengths and weaknesses. Data were obtained in a randomized placebo-controlled study setup that met all criteria for good clinical practice. Nevertheless, the presented analyses are secondary, and the outcome variables were not included in study sample size calculations, and therefore we might have dismissed actual differences between treatment groups. We chose to record food intake retrospectively rather than prospectively to avoid the possibility of the ongoing registration affecting participants' food choices during the registration period. However, the rather long recall period (12 weeks) may also have biased the results. In addition, estimation of energy intake by FFQs is limited by a certain inaccuracy, and finally it should be noted that the applied questionnaire has not been validated in this specific population. Physical activity was also assessed using a nonvalidated scale. Objective measures could have been obtained by use of, for example, accelerometers; however, this would have been at the expense of a greater participant device load, and we therefore decided to rely on patient-reported outcomes.

Liraglutide was administered once daily in this study. Today, an alternative strategy for adding a GLP-1 receptor agonist to insulin therapy would be once-weekly administration. However, at the time of protocol development there was only limited availability of and experience with weekly injections of GLP-1 receptor agonists, especially among people with T1D.

In conclusion, we found that 26 weeks of liraglutide treatment resulted in loss of both fat and lean body mass. Loss of lean body mass was not a consequence of a less physically active lifestyle. Proportionally more fat was lost from android than gynoid depots. Parallel reductions in energy intake between the intervention and placebo groups were found by retrospective registration of food intake; however, food frequency recordings indicated that liraglutide treatment led to a diminished intake of added sugars. All in all, this study 
contributes to existing knowledge with a further characterization of previously demonstrated glycaemic and weight-reducing effects of liraglutide in persons with T1D.

\section{ACKNOWLEDGMENTS}

We thank the participants and research team members Anette Hougaard and Merete Meldgaard Andersen for making this study possible. This study was supported by an unrestricted grant from Novo Nordisk A/S. The funder had no role in any part of this article.

\section{CONFLICT OF INTEREST}

S.S. has served on an advisory panel for Medtronic and has received a research fellowship grant from the Danish Diabetes Academy supported by the Novo Nordisk Foundation. C.S.F. has received research support and lecture fees from Novo Nordisk. T.F.D. has served on advisory panels for Novo Nordisk, has received research support from Novo Nordisk and AstraZeneca and has received lecture fees from Novo Nordisk, AstraZeneca, Sanofi-Aventis and Boehringer-Ingelheim. D.V. is a shareholder in Novo Nordisk. J-E.B.J. has served on advisory panels for Amgen, Eli Lilly, UCB Pharma, and Gedeon Richter, and has received lecture fees from Amgen, Eli Lilly, UCB Pharma, Gilead and Otsuka. S.M. has served on advisory panels for AstraZeneca, Boehringer-Ingelheim, Eli Lilly, Merck Sharp \& Dome, Novo Nordisk, Sanofi and Bayer, has received lecture fees from AstraZeneca, Boehringer-Ingelheim, Merck Sharp \& Dohme, Novo Nordisk and Sanofi, and has received research grants from Novo Nordisk and Boehringer-Ingelheim. H.U.A. is a shareholder in Novo Nordisk, has served on advisory panels for Abbott, Novo Nordisk and AstraZeneca, and has received lecture fees from Nordic Infucare. K.N. is a shareholder in Novo Nordisk, has received research support from Novo Nordisk, Roche Diagnostics, Medtronic and Zealand Pharma, has received lecture fees from Medtronic, Roche Diagnostics, Rubin Medical, Sanofi, Zealand Pharma, Novo Nordisk and Dexcom, and has served on advisory panels for Medtronic, Abbott and Novo Nordisk. T.H. and S.F.O. have nothing to declare.

\section{AUTHOR CONTRIBUTIONS}

Signe Schmidt, Christian Seerup Frandsen, Thomas Fremming Dejgaard, Sten Madsbad, Henrik Ullits Andersen and Kirsten Nørgaard contributed to the study design, data collection and analysis. Kirsten Nørgaard was study sponsor and recipient of the Novo Nordisk A/S study grant. Thórhallur Halldórsson, Sjudur Frodi Olsen and Jens-Erik Beck Jensen contributed to the data collection and analysis. Dorte Vistisen contributed to the data analysis. All authors were involved in the writing of the manuscript and approved the final version of the manuscript.

\section{PEER REVIEW}

The peer review history for this article is available at https://publons. com/publon/10.1111/dom.14567.

\section{DATA AVAILABILITY STATEMENT}

Restrictions apply to the availability of data generated or analysed during this study to preserve patient confidentiality. The corresponding author will, on request, detail the restrictions and any conditions under which access to some data may be provided.

\section{ORCID}

Signe Schmidt (D) https://orcid.org/0000-0002-6968-6675

Thomas F. Dejgaard (D) https://orcid.org/0000-0002-0097-7052

Sten Madsbad (D) https://orcid.org/0000-0002-5017-1815

\section{REFERENCES}

1. Conway B, Miller RG, Costacou T, et al. Temporal patterns in overweight and obesity in type 1 diabetes. Diabet Med. 2010;27(4): 398-404.

2. Foster NC, Beck RW, Miller KM, et al. State of type 1 diabetes management and outcomes from the T1D exchange in 2016-2018. Diabetes Technol Ther. 2019;21(2):66-72.

3. Miller KM, Foster NC, Beck RW, et al. Current state of type 1 diabetes treatment in the U.S.: updated data from the $11 \mathrm{~d}$ exchange clinic registry. Diabetes Care. 2015;38(6):971-978.

4. Vestberg D, Rosengren A, Olsson M, Gudbjörnsdottir S, Svensson A-M, Lind M. Relationship Between Overweight and Obesity With Hospitalization for Heart Failure in 20,985 Patients With Type 1 Diabetes. Diabetes Care. 2013;36(9):2857-2861. https://doi. org $/ 10.2337 / \mathrm{dc} 12-2007$

5. The Diabetes Control and Complications Trial Research Group. The effect of intensive treatment of diabetes on the development and progression of long-term complications in insulin-dependent diabetes mellitus. N Engl J Med. 1993;329(14):977-986.

6. Dixon JB. The effect of obesity on health outcomes. Mol Cell Endocrinol. 2010;316(2):104-108.

7. Edqvist J, Rawshani A, Adiels $\mathrm{M}$, et al. BMI, mortality, and cardiovascular outcomes in type 1 diabetes: findings against an obesity paradox. Diabetes Care. 2019;42(7):1297-1304.

8. Prospective Studies Collaboration. Body-mass index and causespecific mortality in 900000 adults: collaborative analyses of 57 prospective studies. Lancet. 2009;373(9669):1083-1096.

9. Frandsen CS, Dejgaard TF, Madsbad S. Non-insulin drugs to treat hyperglycaemia in type 1 diabetes mellitus. Lancet Diabetes Endocrinol. 2016;8587(16):1-15.

10. Garg SK, Michels AW, Shah VN. Use of non-insulin therapies for type 1 diabetes. Diabetes Technol Ther. 2013;15(11):901-908.

11. Tandon S, Ayis S, Hopkins D, Harding S, Stadler M. The impact of pharmacological and lifestyle interventions on body weight in people with type 1 diabetes: a systematic review and meta-analysis. Diabetes Obes Metab. 2021;23(2):350-362.

12. Avgerinos I, Manolopoulos A, Michailidis T, et al. Comparative efficacy and safety of glucose-lowering drugs as adjunctive therapy for adults with type 1 diabetes: a systematic review and network metaanalysis. Diabetes Obes Metab. 2021;23(3):822-831.

13. Ahren B, Hirsch IB, Pieber TR, et al. Efficacy and safety of liraglutide added to capped insulin treatment in subjects with type 1 diabetes: the adjunct two randomized trial. Diabetes Care. 2016;39(10):16931701.

14. Mathieu C, Zinman B, Hemmingsson JU, et al. Efficacy and safety of liraglutide added to insulin treatment in type 1 diabetes: the adjunct one treat-to-target randomized trial. Diabetes Care. 2016;39(10): 1702-1710.

15. Kuhadiya ND, Dhindsa $S$, Ghanim $H$, et al. Addition of liraglutide to insulin in patients with type 1 diabetes: a randomized placebo-controlled clinical trial of 12 weeks. Diabetes Care. 2016;39:1-9.

16. Frandsen CS, Dejgaard TF, Holst JJ, Andersen HU, Thorsteinsson B, Madsbad S. Twelve-week treatment with liraglutide as add-on to insulin in normal-weight patients with poorly controlled type 
1 diabetes: a randomized, placebo- controlled, double-blind paralle study. Diabetes Care. 2015;38(12):2250-2257.

17. Dejgaard TF, Frandsen CS, Hansen TS, et al. Efficacy and safety of liraglutide for overweight adult patients with type 1 diabetes and insufficient glycaemic control (Lira-1): a randomised, double-blind, placebo-controlled trial. Lancet Diabetes Endocrinol. 2016;4(3): 221-232.

18. Dejgaard TF, Schmidt S, Frandsen CS, et al. Liraglutide reduces hyperglycaemia and body weight in overweight, dysregulated insulinpump-treated patients with type 1 diabetes: the lira pump trial-a randomized, double-blinded, placebo-controlled trial. Diabetes Obes Metab. 2020;22(4):492-500.

19. Ghanim H, Batra M, Green K, et al. Liraglutide treatment in overweight and obese patients with type 1 diabetes: a 26-week randomized controlled trial; mechanisms of weight loss. Diabetes Obes Metab. 2020;22(10):1742-1752.

20. Brock C, Hansen CS, Karmisholt J, et al. Liraglutide treatment reduced interleukin-6 in adults with type 1 diabetes but did not improve established autonomic or polyneuropathy. Br J Clin Pharmacol. 2019; 85(11):2512-2523.

21. Dubé MC, D'Amours M, Weisnagel SJ. Beyond glycaemic control: a cross-over, double-blinded, 24-week intervention with liraglutide in type 1 diabetes. Diabetes Obes Metab. 2018;20(1):178-184.

22. World Health Organisation (WHO). Waist Circumference and WaistHip Ratio. Geneva, Switzerland: Report of a WHO Expert Consultation; 2008 http://www.who.int

23. Mikkelsen TB, Olsen SF, Rasmussen SE, Osler M. Relative validity of fruit and vegetable intake estimated by the food frequency questionnaire used in the Danish National Birth Cohort. Scand J Public Health. 2007;35(2):172-179.

24. Mikkelsen TB, Osler M, Olsen SF. Validity of protein, retinol, folic acid and $n-3$ fatty acid intakes estimated from the food-frequency questionnaire used in the Danish National Birth Cohort. Public Health Nutr. 2006;9(6):771-778.

25. Olsen SF, Mikkelsen TB, Knudsen VK, et al. Data collected on maternal dietary exposures in the Danish National Birth Cohort. Paediatr Perinat Epidemiol. 2007;21(1):76-86.

26. National Food Institute DTU Foods public food database. 2019. https://frida.fooddata.dk/?lang=en. Accessed September 9, 2021.

27. Jendle J, Nauck MA, Matthews DR, et al. Weight loss with liraglutide, a once-daily human glucagon-like peptide-1 analogue for type 2 diabetes treatment as monotherapy or added to metformin, is primarily as a result of a reduction in fat tissue. Diabetes Obes Metab. 2009; 11(12):1163-1172.

28. Berentzen T, Sørensen TIA. Effects of intended weight loss on morbidity and mortality: possible explanations of controversial results. Nutr Rev. 2006;64(11):502-507.

29. Bigaard J, Frederiksen K, Tjønneland A, et al. Body fat and fat-free mass and all-cause mortality. Obes Res. 2004;12(7):1042-1049.
30. Wiklund $P$, Toss $F$, Weinehall $L$, et al. Abdominal and gynoid fat mass are associated with cardiovascular risk factors in men and women. J Clin Endocrinol Metab. 2008;93(11):4360-4366.

31. Huxley R, Mendis S, Zheleznyakov E, Reddy S, Chan J. Body mass index, waist circumference and waist:hip ratio as predictors of cardiovascular risk-a review of the literature. Eur J Clin Nutr. 2010;64(1):16-22.

32. Seidell JC. Waist circumference and waist/hip ratio in relation to allcause mortality, cancer and sleep apnea. Eur J Clin Nutr. 2010;64(1): 35-41.

33. Sarwer DB, Polonsky HM. The psychological burden of obesity. Endocrinol Metab Clin North Am. 2016;45(3):677-688.

34. Freedman LS, Commins JM, Moler JE, et al. Pooled results from 5 validation studies of dietary self-report instruments using recovery biomarkers for energy and protein intake. Am J Epidemiol. 2014; 180(2):172-188.

35. Shah M, Vella A. Effects of GLP-1 on appetite and weight. Rev Endocr Metab Disord. 2014;15(3):181-187.

36. Holst JJ. The physiology of glucagon-like peptide 1. Physiol Rev. 2007;87(4):1409-1439.

37. Horowitz $M$, Flint $A$, Jones $K L$, et al. Effect of the once-daily human GLP-1 analogue liraglutide on appetite, energy intake, energy expenditure and gastric emptying in type 2 diabetes. Diabetes Res Clin Pract. 2012;97(2):258-266.

38. Blundell J, Finlayson G, Axelsen M, et al. Effects of once-weekly semaglutide on appetite, energy intake, control of eating, food preference and body weight in subjects with obesity. Diabetes Obes Metab. 2017;19(9):1242-1251.

39. Thomas D, Elliott EJ. Low glycaemic index, or low glycaemic load, diets for diabetes mellitus. Cochrane Database of Systematic Reviews. 2009;(1):1-28. https://doi.org/10.1002/14651858.cd006296.pub2

40. Russell-Jones D, Khan R. Insulin-associated weight gain in diabetes -causes, effects and coping strategies. Diabetes Obes Metab. 2007; 9(6):799-812.

41. Mella R, Schmidt CB, Romagnoli P-P, Teske JA, Perez-Leighton C. The food environment, preference, and experience modulate the effects of Exendin-4 on food intake and reward. Obesity (Silver Spring). 2017;25(11):1844-1851.

42. Kadouh H, Chedid V, Halawi H, et al. GLP-1 analog modulates appetite, taste preference, gut hormones, and regional body fat stores in adults with obesity. J Clin Endocrinol Metab. 2020;105(5):1552-1563.

How to cite this article: Schmidt S, Frandsen CS, Dejgaard TF, et al. Liraglutide changes body composition and lowers added sugar intake in overweight persons with insulin pump-treated type 1 diabetes. Diabetes Obes Metab. 2022;24(2):212-220. doi:10.1111/dom.14567 